\title{
A Protocol-Driven, Digital Conversational Agent at the Hospital Bedside to Support Nurse Teams and to Mitigate Delirium and Falls Risk
}

Victor Wang ${ }^{1}, \mathrm{SM}$; Sharon Wexler ${ }^{2}, \mathrm{PhD}, \mathrm{RN}$; Lin Drury ${ }^{2}, \mathrm{PhD}, \mathrm{RN}$; Brittany $\mathrm{Wang}^{1}, \mathrm{MA}$

1 care.coach corporation, Millbrae, CA, United States

${ }^{2}$ Pace University, New York, NY, United States

Corresponding Author:

Victor Wang, SM

care.coach corporation

660 El Camino Real

Millbrae, CA,

United States

Phone: 8666964

Email: victor@care.coach

\section{Abstract}

Background: Up to 1 million falls per year occur during hospital stays in the US, costing hospitals up to $\$ 7$ billion per year to treat. Delirium, a major cause of falls, is highly prevalent (29\%-64\%) among hospitalized elders. Our team is developing a care technology that provides $24 \times 7$ proactive patient support to mitigate falls and delirium in a scalable, cost-effective, yet person-centered manner. We were awarded an NIH SBIR Fast-Track grant to further our work.

Objective: Prior research showed that digital avatars can build psychosocially supportive relationships with older adults living in the community. We conducted a pilot study of these digital avatars in a community hospital setting, and found that bedside avatars helped reduce the rate of falls among elderly inpatients while mitigating delirium and loneliness. Thus, in our present NIH-funded work, we seek to enhance the clinical robustness of our avatar intervention by implementing a more complete set of protocols derived from the evidence-based Hospital Elder Life Program (HELP), and to validate the benefits of this next-generation intervention in a larger scale, multi-site clinical study.

Methods: Avatars are displayed on tablets as virtual dogs or cats, responding to touch. They are able to talk intelligently due to live support from a $24 \times 7$ team of trained humans combined with protocol guidance and process automation software. At the bedside, avatars talk with patients and implement protocols including reorientation and bed exercises, based on HELP. English-speaking patients aged 65+ on three medical surgical units were selected in our pilot study based on subjective nursing risk assessment and patient consent. Participants on two intervention units received an avatar for their entire hospital stay. Participants on a control unit received a daily visit from a nursing student. Measures were administered upon study enrollment and discharge. Quarterly fall rates by unit were reported by the hospital. In our present work, we will conduct a larger, multi-site clinical study starting with two hospitals in late 2018 and incorporating electronic medical record integration to increase the objectivity of patient enrollment, ease the patient enrollment process, and apply interventions intelligently tailored to each patient. Additional hospitals may join the two-year study through 2020.

Results: In our pilot study, intervention $(n=41)$ and control $(n=54)$ patients were equivalent except for an intervention group bias toward females $(P=.02)$. Mean age was 76.5 years, with $55 \%$ female, $46 \%$ African-American, and $44 \%$ speaking English as a second language. Typical length of stay was 3-6 days. On average, avatars performed 71.3 observational check-ins, 61 minutes of engagement using 11.5 images or audio files, and 6.5 protocol-driven tasks per patient per day. Compared to control patients, intervention patients showed a significantly greater reduction in delirium score (CAM, $P=.003$ ) and loneliness (UCLA-LS, $P=.008$ ). The primary intervention unit had 0.9 falls per 1000 -patient days during the 3 -month study period, compared to 6.5 on the control unit (86\% reduction).

Conclusions: Previous findings support the use and development of avatar technology for hospitalized patients at risk for delirium or falls. Hospitals interested in joining our two-year clinical study may contact the principal investigator.

(iproc 2018;4(2):e11883) doi: $\underline{10.2196 / 11883}$ 


\section{KEYWORDS}

avatars; clinical trials; elderly; health care technology; hospital information systems; loneliness; mobile health; mHealth; patient care; psychosocial support systems; falls; delirium; nursing; Hospital Elder Life Program; Confusion Assessment Method; HELP; hospitals; SBIR

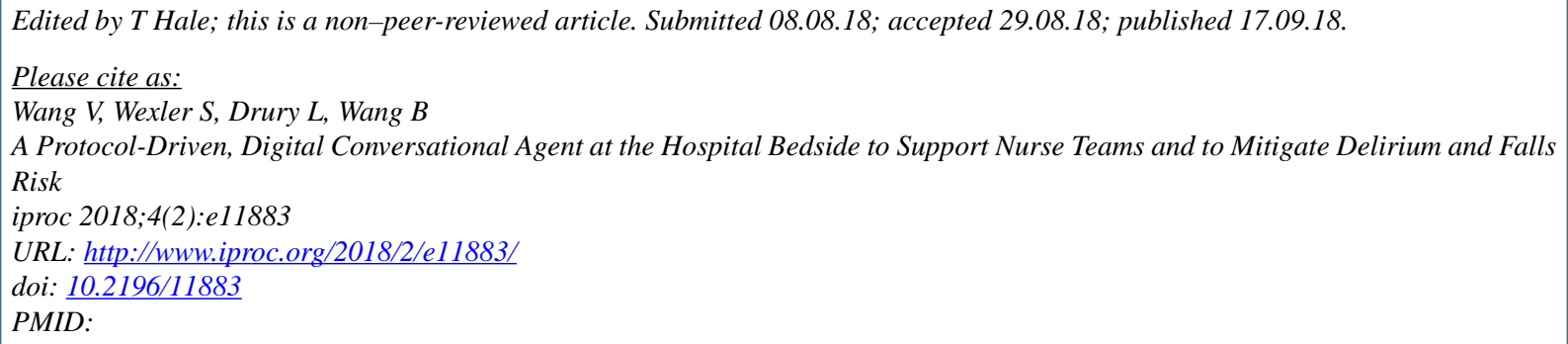

(C) Victor Wang, Sharon Wexler, Lin Drury, Brittany Wang. Originally published in Iproceedings (http://www.iproc.org), 17.09.2018. This is an open-access article distributed under the terms of the Creative Commons Attribution License (https://creativecommons.org/licenses/by/4.0/), which permits unrestricted use, distribution, and reproduction in any medium, provided the original work, first published in Iproceedings, is properly cited. The complete bibliographic information, a link to the original publication on http://www.iproc.org/, as well as this copyright and license information must be included. 